Spectroscopy of Argon Excited in an Electron Beam Ion Trap

E. Trabert

April 22, 2005

Comments of Atomic, Molecular, and Optical Physics 
This document was prepared as an account of work sponsored by an agency of the United States Government. Neither the United States Government nor the University of California nor any of their employees, makes any warranty, express or implied, or assumes any legal liability or responsibility for the accuracy, completeness, or usefulness of any information, apparatus, product, or process disclosed, or represents that its use would not infringe privately owned rights. Reference herein to any specific commercial product, process, or service by trade name, trademark, manufacturer, or otherwise, does not necessarily constitute or imply its endorsement, recommendation, or favoring by the United States Government or the University of California. The views and opinions of authors expressed herein do not necessarily state or reflect those of the United States Government or the University of California, and shall not be used for advertising or product endorsement purposes. 


\title{
Spectroscopy of argon excited in an electron beam ion trap
}

\author{
E. Träbert \\ Experimentalphysik III, Ruhr-Universität Bochum, D-44780 Bochum, Germany, and \\ High Temperature and Astrophysics Division, Physics and Advanced Technologies, \\ Lawrence Livermore National Laboratory, Livermore, CA 94550-9234, U.S.A.*
}

(Dated: April 17, 2005)

\begin{abstract}
Argon is one of the gases best investigated and most widely used in plasma discharge devices for a multitude of applications that range from wavelength reference standards to controlled fusion experiments. Reviewing atomic physics and spectroscopic problems in various ionization stages of Ar, the past use and future options of employing an electron beam ion trap (EBIT) for better and more complete Ar data in the x-ray, EUV and visible spectral ranges are discussed.
\end{abstract}

PACS numbers: $32.30 . J_{c}, 34.50 . F a, 32.70 . \mathrm{Cs}$

\section{INTRODUCTION}

Spectroscopic light sources have been developed in the last 150 years that range from flames, arcs, sparks, tokamak (low-density) and laser-produced (high-density) plasmas, foil-excited ion beams (high-density excitation, time-resolved observation in a low-density environment) to electron beam ion traps, and that open up all ionization stages of all elements for spectroscopic study. The latest of the aforementioned devices, the electron beam ion trap (EBIT), is now almost 20 years of age. It has a number of peculiar properties that are particularly interesting for certain spectroscopic investigations. Among these are the low kinetic energy of the trapped ions, avoiding the need for Doppler shift correction, and the low particle density that permits the study of spontaneous radiative decays of atomic levels with lifetimes in the range of milliseconds to seconds. Such time constants are typical for magnetic dipole (M1) and similar electric-dipole forbidden transitions that are of interest in fundamental atomic structure studies, in astrophysical observations and for the diagnostics of fusion test plasma devices $[1,2]$.

Argon is the most abundant rare gas in the atmosphere, and, as a monatomic gas, it is also frequently used in a wide variety of plasma discharges. One of the latest uses of argon is that of a radiation blanket in controlledfusion oriented machines. For this application, spectral information on the light emission in all wavelength ranges is essential. The web-oriented data base of the US National Institute of Standards and Technology (NIST) lists about 900 lines of Ar in many charge states in the wavelengths ranges from the soft-x-ray range to the near infrared at $850 \mathrm{~nm}$ [3]. Certainly much more information is available in the literature (see, for example, [4]), though apparently not always of a quality sufficient to merit acceptance into the NIST tables.

However, there also are surprises that result from the choice of light sources. For example, there are stud-

*Electronic address: traebert@ep3.rub.de ies of various sections of the argon spectrum that employ foil-excited fast ion beams, and there are studies of argon under ion beam irradiation [5, 6], and the richness in spectral lines has defied complete analysis for either light source. Moreover, the spectra clearly differ for prompt (within a few nanoseconds) or delayed light emission (within a few hundred ns) after an excitation pulse, just as delayed beam-foil spectra (recorded while observing the ion beam at a position a few $\mathrm{mm}$ or $\mathrm{cm}$ after leaving the exciter foil, and thus some $100 \mathrm{ps}$ to a dozen ns after excitation) $[7,8]$ are very different in appearance from prompt ones. Consequently time-resolution can be a very valuable tool in spectroscopy, and low-density light sources that permit studies on the millisecond time scale may reveal spectral information that cannot be gained from standard light sources in which collisions quench long-lived levels within nano- or at best microseconds. Low particle density is also a key to obtain laboratory spectra that resemble astrophysical spectra.

I take the opportunity to survey atomic physics problems that relate to the various charge states of argon, many of which have already been studied using electron beam ion traps. I discuss what the advantage is of employing an electron beam ion trap, a preeminent lowdensity light source, and why it is a promising choice for further experiments - not only on argon.

\section{EXPERIMENTAL PROCEDURES WITH ELECTRON BEAM ION TRAPS}

The first electron beam ion traps (EBIT-I, SuperEBIT and EBIT-II) [9, 10] have been constructed at the University of California Lawrence Livermore National Laboratory (LLNL). In an electron beam ion trap, atoms are ionized by electron-atom collisions and then trapped. While being trapped in a combination of electric and magnetic fields, the "stationary" ions are further bombarded by the same electron beam. This is kinematically inverse to the process of excitation in ion-accelerator experiments in which a fast ion beam is being passed through a thin foil that acts as a stationary electron target. SuperEBIT can reach electrons energies of up 
to $250 \mathrm{keV}$, which is sufficient to completely ionize uranium, whereas a fast uranium ion beam needs acceleration to about $500 \mathrm{MeV} /$ nucleon to reach the same bare ion state [11]. SuperEBIT can also run at very low electron energies (below $100 \mathrm{eV}$ ) in the trap region [12], by adjustment of the drift tube voltages independently of the floating electron gun voltage. For most of the work on Ar, electron energies between $80 \mathrm{eV}$ and $20 \mathrm{keV}$ are used, with electron beam currents up to $100 \mathrm{~mA}$ at the higher energies. The intense electron beam is guided and compressed to about 60 to $70 \mu \mathrm{m}$ diameter $[10,13]$ by a strong (3 to $5 \mathrm{~T}$ ) magnetic field.

Argon atoms are injected into an electron beam ion trap through a gas injector, either pulsed [14] or steadystate. The ultra-high vacuum (UHV) gas plume intersects the electron beam. Any ions formed are then attracted by the space charge of the electron beam, being also confined radially by the magnetic field and axially by suitable electric potential steps. Since the electron beam intersects the trap volume, further electron-ion collisions take place, and stepwise ionization and some recombination take place. Under appropriate vacuum conditions, the highest charge state reached of any element is simply determined by the ionization potential of that ion species (Fig. 1). This implies that by lowering the electron beam energy below a certain threshold (the ionization potential of the next-lower charge state ion), a given charge state (and all higher ones) can be suppressed. Such selectivity is very helpful for the analysis of multi-line spectra $[15,16]$.

Continuous gas injection, however, has side effects. As neutral atoms are supplied all the time and then ionized, ions of all charge states up to the maximum are present, and the operating conditions may shift the charge state balance, but without fully suppressing low charge state ions. The same intermediate charge state ions can thus be produced either as near-maximum charge state ions reached at relatively low electron beam energies (under good vacuum conditions), or as transient products when running at high electron beam energies. The latter case regularly permits to operate at higher electron beam currents, which in turn yields a higher signal rate (proportional to both the electron beam current $I_{e}$ and the density of ions and thus to $I_{e}^{2}$ ), but at the cost of clean operating conditions. For example, in time-resolved measurements, the recombination of ions in higher charge states can play a detrimental role [17].

For measurements of long atomic lifetimes ( $\mu$ s to many $\mathrm{ms}$ ), the electron beam energy can either be modulated around the production threshold of the level of interest or can be fully switched off and on [18-20]. In the latter mode of operation, the ions remain confined by the electron beam ion trap in its magnetic trapping mode $[18,21]$ where operation corresponds to that of a Penning trap. For the measurement of very short level lifetimes (fs range), the ion temperature in the trap can be lowered so much that the Doppler width of a spectral line can be smaller than the line width associated with the uncertainty principle [22].

\section{PHYSICS TOPICS AND ISOELECTRONIC SEQUENCES}

\section{A. H-like Ar XVIII}

One usually assumes of one-electron (hydrogen-like) ions that it is possible to calculate their structure precisely and reliably, so that very precise measurements can test intricate contributions to atomic structure, in particular the radiative or quantum electrodynamic (QED) contributions, most notably the Lamb shift. With the aim of determining the $2 \mathrm{~s}$ Lamb shift, the term separation of the $2 \mathrm{~s}_{1 / 2}$ and $2 \mathrm{p}_{1 / 2}$ levels, fast $\mathrm{Ar}^{17+}$ ion beams have been passed through magnetic fields so that the motional electric fields would Stark-mix the $n=2$ levels. This effected a progressive shortening of the $2 \mathrm{~s}$ level lifetime with increasing $B$ field strength, from which the zero-field level separation could be determined with a precision of about $1 \%$ [23].

Because of the $n^{-3}$ scaling of the Lamb shift as a function of principal quantum number $n$, the $n=1$ Lamb shift is a factor of eight larger than the $n=2$ Lamb shift, but to measure it requires a more precise measurement of a large entity, the $n=1-2$ transition energy. Fast-ion beams have been employed in this quest first $[24,25]$, but the measurements were limited in accuracy by the Doppler correction necessary in observations of fast-moving emitters. X-ray energies near $3.3 \mathrm{keV}$ (Ar XVIII $\mathrm{Ly}_{\alpha}$ ) were determined to within $0.5 \mathrm{eV}$, which is about half of the predicted ground-state Lamb shift. The next step was another fast beam of highly charged heavy ions, but it was not observed directly; it served to produce much slower Ar recoil ions by ion-atom collisions in an Ar gas target [26, 27]. While the Doppler effect could be drastically reduced this way, the gas target provided plenty of collision partners from which the freshly produced recoil ions could capture electrons, resulting in the appearance of satellite lines. The strongest of these satellite lines, from electrons in $n=2$ levels, were modeled and then the model spectra subtracted from the raw spectra. The remaining spectral lines were still asymmetric because of satellites from higher-n electrons, a contribution that then was treated by fitting line profiles to the data. From a $5 \mathrm{ppm}$ wavelength measurement the authors derived the $n=1$ Lamb shift with a precision of $1.5 \%$ - an improvement by a factor of 30 over the preceding fast-ion beam work.

However, the multi-step correction for satellite line contamination is not beyond doubt, and measurements in a higher-vacuum environment might be able to reduce the problem. One approach has been using a tokamak plasma device [28], but only an $n=2$ Lamb shift value to no better than $3 \%$ has been reported from this experiment. A more precise and accurate value may be expected from an electron beam ion trap, because the charge state distribu- 
tion can be varied in order to check for systematic errors associated with satellite lines. Moreover, there is no notable Doppler shift, the excitation processes do not favour core-excited states, and the vacuum is extremely good and thus discourages many-body collisions that might lead to recombination and the population of high-lying levels. An experiment to this aim has been started at the Oxford EBIT. The limiting problem in such precision work is that of reference wavelengths. Many of the earlier $\mathrm{x}$-ray reference wavelength data have been found to be less accurate than believed at the time, and practically any newly measured element requires the calibration effort of a reference light source first. One elegant way out is the precise determination of the crystal lattice spacing of a crystal interferometer, a device that then permits the direct determination of absolute wavelengths. This technique has been demonstrated on H-like magnesium and silicon ions stored in a Livermore electron beam ion trap [29-31], but it has not yet been applied to H-like argon ions.

In any case, it will be difficult to challenge QED theory [32]. In uranium ( $\mathrm{Z}=92$ ), theory for the $n=1$ level Lamb shift (of roughly $4 \mathrm{keV}$ ) claims to be accurate to a fraction of $1 \mathrm{eV}$, while the most precise experiments fight to reach the $10 \mathrm{eV}$ level. More precise are several measurements of the $n=2$ Lamb shift in Li-like $\mathrm{U}$, with an uncertainty of $0.1 \mathrm{eV}[33,34]$ (out of $280 \mathrm{eV}$ total), and even more precise results are expected from an ongoing experiment at SuperEBIT [35]. The total Lamb shift is much smaller in Ar. However, laser spectroscopic experiments on several elements lighter than Ar [36] all fall to one side of the predictions, and it would be interesting to see whether this trend continues to Ar and whether it signals a missing term in the calculations that might be notable only in this range. It is for this reason that one of the key experiments envisioned for the Oxford EBIT was a laser spectroscopic study of a hydrogen-like ion ( $\mathrm{Si}^{13+}$ for reasons of the available laser) [37].

\section{B. He-like Ar XVII}

For ground-state transitions in He-like Ar, the same techniques as for the study of H-like ions, for example the fast-ion beam technique [25], can be used and have been used. However, the strong lines associated with the $1 \mathrm{~s}^{2}{ }^{1} \mathrm{~S}_{0}-1 \mathrm{~s} 2 \mathrm{p}{ }^{1,3} \mathrm{P}_{1}^{\circ}$ transitions have not only the $1 \mathrm{~s}^{2}{ }^{1} \mathrm{~S}_{0}$ - $1 \mathrm{~s} 2 \mathrm{p}{ }^{3} \mathrm{P}_{2}^{\circ}$ and $1 \mathrm{~s}^{2}{ }^{1} \mathrm{~S}_{0}-1 \mathrm{~s} 2 \mathrm{~s}{ }^{3} \mathrm{~S}_{1}$ lines nearby (the $\mathrm{w}$, $\mathrm{y}, \mathrm{x}$, and $\mathrm{z}$ lines, respectively, of plasma spectroscopy in the $\mathrm{x}$-ray range [38]), but also a plethora of satellite lines from core-excited levels in Li-, Be- and B-like ions. The efficient population of such core-excited levels in the fastion - foil interaction renders beam-foil spectroscopy unsuitable for precision spectroscopy of the resonance lines in He-like ions - there are too many satellite lines near by. Possibly electron capture by hydrogen-like ions in a dilute gas target could be exploited to reduce this problem, but an electron beam ion trap, with its very differ- ent level population mechanism that disfavours multiply excited levels, would probably be the better choice, with the added benefit of avoiding the need for Doppler corrections. A study of the satellite lines has very recently been reported from the Oxford EBIT [39], but in these measurements the diagram lines in the He-like ion, though imperfectly known, served as wavelength references. Of course, x-ray spectra of Ar can be evaluated towards a determination of QED contributions, too [40].

In the vacuum ultraviolet region, He-like ions offer access to measurements of the $n=2$ Lamb shift. The advantage over measurements on one-electron ions is the much longer lifetime of the $n=2$ triplet levels (compared to singlet levels or the doublet levels in H-like ions), because the ground state decay channel is largely blocked in not-too heavy ions. Consequently spectroscopy can deal with lines (from $n=2, \Delta n=0$ transitions) that are not notably lifetime-broadened. The drawback of this atomic system is the need for precise calculations of a two-electron ion. Meanwhile the QED calculations have reached a level of development at which they are considered to be more accurate than the non-QED part of the calculations of multi-electron ions. Thus precise measurements are still very much needed, but less so for the testing of QED contributions than for testing the dominant, non-QED quantum mechanical part.

He-like $\mathrm{Ar}^{16+}$, however, has been a problem child of spectroscopy. Of the three $n=2$ triplet levels of interest, the $1 \mathrm{~s} 2 \mathrm{p}^{3} \mathrm{P}_{1}^{\circ}$ level decays predominantly (by spinchanging electric dipole (E1) transition) to the ground state, $1 \mathrm{~s}^{2}{ }^{1} \mathrm{~S}_{0}$. The $1 \mathrm{~s} 2 \mathrm{p}{ }^{3} \mathrm{P}_{2}^{\circ}$ level decays with about equal branches to the ground state (M2 transition) and to the lowest triplet level, $1 \mathrm{~s} 2 \mathrm{~s}{ }^{3} \mathrm{~S}_{1}$. The remaining level, $1 \mathrm{~s} 2 \mathrm{p}{ }^{3} \mathrm{P}_{0}^{\circ}$, cannot decay by emission of a single photon to the ground state and (practically) decays to the $1 \mathrm{~s} 2 \mathrm{~s}$ ${ }^{3} \mathrm{~S}_{1}$ level only. Consequently, one expects two roughly equally strong (or rather weak) lines in the VUV to represent the $2 \mathrm{~s}-2 \mathrm{p}$ transition array in Ar XVII. The lines have been reported from recoil ion spectra [41, 42], but fast-ion beam spectra [43] later on have cast doubt on the validity of the earlier reports. The authors of the later study see their experiment not as just an improvement in precision, but rather as a fundamental improvement identifying a different line than before with the $\mathrm{J}=0$ level decay, in better agreement with their own isoelectronic analysis in comparison to theory. They imply that, in the preceding studies, the lines sought for may have been too weakly excited to be identified properly against the ubiquitous lines of other (unclear) origin in any gas target under ion irradiation. An electron beam ion trap with its controllable charge state distribution, absence of Doppler shifts, and with a much lower background pressure, might be helpful in providing a 'second opinion'.

In the $\mathrm{x}$-ray range, the $\mathrm{K}_{\alpha}$ and $\mathrm{K}_{\beta}$ lines, their relative intensities, polarization parameters, satellite lines, line profiles and whatever else are being studied in order to learn about fusion test plasmas [44, 45]. However, much of the plasma diagnostical evaluation depends on theo- 
retical modeling, and it would certainly be advantageous to test such modeling on a simpler situation than that encountered in a tokamak or plasma pinch experiment. Obviously, the better vacuum and simpler geometry of the electron beam ion trap offers just that, and a number of electron beam ion trap studies have already investigated these very lines of argon [46-51]. One of these studies of the $\mathrm{K}_{\beta}$ lines employed an electron beam ion trap (at Troitsk, Russia) that works with regular (not superconducting) electromagnets providing a magnetic field of about $0.65 \mathrm{~T}$. Also, the vacuum is then worse than in the cryogenic devices $\left(\mathrm{p} \approx 3-5 \cdot 10^{-9}\right.$ Torr [50]). This did not hurt this particular experiment, since inner-shell vacancies in Ar II and Ar III, that is, in singly and doubly charged ions of argon, were studied. Interestingly, this experiment required pulsed operation of the electron beam ion trap, in particular of the electron beam, because a steady electron beam would have removed the outer electrons that were of interest here. The unresolved $\mathrm{K}_{\beta}$ line profiles were analyzed with the aid of Cowan code (Hartree-Fock) calculations.

The Ar x-ray spectrum in the region of the prominent $n=1$ to $n^{\prime}=2$ lines in He-like ions has been observed with high resolution at the Princeton NSTX tokamak and at the Livermore EBIT-II electron beam ion trap [52]. While the diagram lines stand out, most of the satellite lines that are widely employed for temperature and density diagnostics in various plasma discharges are present as well. There is an amusing facet to this: The satellite labeling scheme that has been introduced by Gabriel [38] names 26 diagram and satellite lines in He- and Li-like ions, one for each letter of the alphabet. This scheme, however, leaves out the M2 decay of the $1 \mathrm{~s} 2 \mathrm{~s} 2 \mathrm{p}{ }^{4} \mathrm{P}_{5 / 2}^{\circ}$ level to the $1 \mathrm{~s}^{2} 2 \mathrm{~s}{ }^{2} \mathrm{~S}_{1 / 2}$ level. While this level has been studied extensively by beamfoil spectroscopic techniques, observing both the M2 and the competing Auger decay branch (see [53] for the work on Ar), the plasma community appears to have relied on the completeness of Gabriel's label list. The M2 line was seen, but was regularly subsumed as a satellite relating to Be-like ions, as has been pointed out after revisiting this line at the Princeton NSTX tokamak and the Livermore electron beam ion trap [52]. If only Gabriel had used a 27-letter alphabet ...

One of the distinct lines in the X-ray spectrum of Helike ions (Gabriel's line $\mathrm{z}$ ) signals the decay of the lowest triplet level, $1 \mathrm{~s} 2 \mathrm{~s}{ }^{3} \mathrm{~S}_{1}$, that is notable only in lowdensity plasmas where the radiative decay rate exceeds the collisional quench rate [54]. The decay rate can be calculated as a purely relativistic process, and a great many attempts have been made to test the calculations by precise experiments. For elements much heavier than argon, beam-foil spectroscopy [55] is the only method presently available, but the precision so far has usually been worse than a few percent (for references, see [5658]). For lighter elements, the Heidelberg heavy-ion storage ring and the Livermore electron beam ion trap have yielded lifetime results with uncertainties as low as $0.5 \%$
[59, 60]. Much work historically has dealt with argon, overcoming the massive systematic errors suffered in the pioneering fast-ion beam work on Ar [61] in the lifetime range near $200 \mathrm{~ns}$. Apparently the best atomic lifetime results for the $1 \mathrm{~s} 2 \mathrm{~s}{ }^{3} \mathrm{~S}_{1}$ level in $\mathrm{Ar}^{16+}$ reached an uncertainty of about 5\% [62] with a slow ion beam. However, at the present level of calculational quality, this precision falls short of testing theory by at least one order of magnitude. The Livermore electron beam ion trap group has tried to reach this nanosecond lifetime range by rapid switching of the electron beam in EBIT-II, but systematic error problems so far precluded meaningful atomic lifetime results in the sub- $\mu$ s range by this technique.

At the Dresden EBIT (a room temperature electron beam ion trap), x-ray spectra of He-like Ar have been used to investigate trap properties and ion production [63]. The latter process benefitted from the addition of light coolant gases like $\mathrm{He}$ and $\mathrm{Ne}$, as is well known for the optimization of trapping times. At the Berlin EBIT, the characteristic line emision and the line ratios of satellite lines were analyzed as a means to further the diagnostics of hot plasmas [64].

\section{Li-like Ar XVI}

Ions with a single electron outside a closed-shell electron core often feature prominent resonance lines so that these charge states are easily recognized. Theory supposedly can calculate the structure and transition rates of such systems to high precision. The good experimental and calculational access invites cross-checks and competition along the isoelectronic sequence.

Edlén [66] cites solar observations [67] as a reference for his semiempirical analysis that yields wavelengths of $35.3866 \mathrm{~nm}$ and $38.9075 \mathrm{~nm}$ for the $2 \mathrm{~s}-2 \mathrm{p}$ resonance lines in Li-like $\mathrm{Ar}^{15+}$. However, more than 20 years later, the NIST Atomic Spectra Database on the WorldWideWeb [3] has no wavelength entry on these lines at all. It is not clear whether this fact implies doubts on the validity of the early data or only funding shortages at the NIST Data Center. An electron beam ion trap (or a tokamak) may be the light source of choice for a future precise laboratory wavelength determination of the resonance lines of Ar XVI. Accurate theoretical wavelength data have been provided by Kim et al. [68].

The resonance transition rates and their inverse, the lifetimes of the $2 p$ levels of Li-like ions, are of interest in testing quantum mechanic algorithms. For $\mathrm{Ar}^{15+}$, the level lifetimes are in the range of a few hundred picoseconds [72]. This is a range that is very convenient for beam-foil spectroscopy. This isoelectronic sequence has been studied up $\operatorname{Kr}(\mathrm{Z}=36)$ [69] where the level lifetime is about 50ps. The latest such exercise has studied Li-like $\mathrm{Ni}$ ions [70]. 


\section{M1 transitions in Be-like Ar XV, B-like} Ar XIV, O-like Ar XI, and F-like Ar X

In the visible-range survey spectrum of Ar as recorded at SuperEBIT (Fig. 2), four Ar lines stand out among the few strongest in the spectrum, in contrast to the hundreds of tabulated known Ar lines that appear in other laboratory light sources (where these four lines usually do not show). These four are transitions with very low transition probabilities that are regularly quenched by collisions in any environment that has collision rates of a few hundred per second or more. These lines arise from electric-dipole forbidden transitions in multiply charged Ar ions and appear brightly in the solar corona and similarly dilute astrophysical plasmas [1,71], and they evidently do the same in the electron beam ion trap, confirming the spectacular applicability of the latter device to many astrophysical problems.

These magnetic dipole (M1) transitions (often with a weak electric quadrupole (E2) admixture) connect levels within the same electron configuration, that is with the same parity, that cannot be connected by E1 transitions. Such low-rate transitions become observable only when no competing faster decay drains the population of the upper level. For example, there certainly are such $\mathrm{M} 1 / \mathrm{E} 2$ transitions between the $2 \mathrm{p} \mathrm{J}=1 / 2,3 / 2$ levels of Li-like ions, but the $2 \mathrm{~s}-2 \mathrm{p}$ E1 transitions are faster by many orders of magnitude so that the transitions between the fine structure levels of the excited term are practically unobservable. In the next more complex system, Be-like ions, there are the rather long-lived $2 \mathrm{~s} 2 \mathrm{p}^{3} \mathrm{P}_{J}^{\circ}$ triplet levels. The $\mathrm{J}=0$ level cannot decay (by single photon emission) to the $\mathrm{J}=0$ ground state. The next higher, $\mathrm{J}=1$, level has an M1 decay to the $\mathrm{J}=0$ level of the same configuration, but the E1 intercombination decay to the ground state is much more probable, even as it requires a change of spin. The $\mathrm{J}=2$ level is the most interesting one in this context. For light ions, the magnetic quadrupole (M2) decay to the ground state dominates, but for Belike ions beyond a nuclear charge of about $\mathrm{Z}=12$, the M1 decay to the $\mathrm{J}=1$ level is strongest $[73,74]$. (An E2 decay branch to $\mathrm{J}=0$ also exists, but remains weak.) A lifetime measurement of the $2 \mathrm{~s} 2 \mathrm{p}^{3} \mathrm{P}_{2}^{\circ}$ level thus yields essentially the inverse of an M1 transition rate in an excited configuration (see below).

Ground configurations usually are more highly populated than excited configurations, and the level structure and transition rates also more easily calculated with precision. There are many such intra-configuration, E1forbidden transitions in the $n=2$ ground configurations of B-like to F-like ions. Of these the B-like and F-like ions are of practical interest, because only a single transition each is possible in these ions $\left(2 \mathrm{~s}^{2} 2 \mathrm{p}^{2} \mathrm{P}_{1 / 2,3 / 2}^{\circ}\right.$ and $2 \mathrm{~s}^{2} 2 \mathrm{p}^{5}$ ${ }^{2} \mathrm{P}_{3 / 2,1 / 2}^{\circ}$, respectively), which implies that all the available intensity will be concentrated in a single line that is more easily observed than the multitude of individually weaker lines in the other ions. Because of this advantage, the M1 transitions in these two ion species were among the first recognized and their wavelengths parameterized by Edlén [75-77], and they are included in the comprehensive survey of forbidden transitions compiled by Kaufman and Sugar [78].

There are four prominent M1 lines in the visible spectrum of Ar. After observation of these lines in the Livermore EBIT [19, 79], the first precise laboratory wavelength measurement of any of these four lines was achieved at the Oxford EBIT [80]. Meanwhile highly precise wavelength measurements of all four Ar M1 lines have been reported by the Heidelberg EBIT group [84]. Three of these lines have been the subject of transition rate measurements using ion traps, including the electron beam ion traps at NIST Gaithersburg, Oxford and Livermore [19, 81-83].

In hindsight, the lifetime results of the first attempt with the NIST EBIT [82] and of experiments using an electrostatic ion trap [83] appear to have suffered from unrecognized errors. For example, in the NIST experiments the signal was found to be best at electron beam energies far beyond (several keV) the nominal production threshold (which is well below $1 \mathrm{keV}$ ). By Livermore experience (where good signal is available at a few hundred $\mathrm{eV}$ above threshold) this suggests less-than-optimum vacuum conditions. The same problem may be expected to yield an apparent lifetime that is shorter than the true one because of ions losses due to charge exchange. Again, an investigation of systematic error sources at Livermore has demonstrated this detrimental effect and how to evaluate it. The Oxford EBIT and the Livermore EBIT-II lifetime results on argon $[19,81]$ are consistent with each other and with data for other elements in the same isoelectronic sequences (from the Livermore electron beam ion traps and from a heavy-ion storage ring) [85-87]. They corroborate mainstream theoretical predictions (after semiempirical correction for experimental term differences). In fact, theory has the problem of estimating reliability intervals for the calculated values, and typical estimates used to run to $10 \%$ uncertainty and more. Now the LLNL EBIT-II and the heavy-ion storage ring measurements show that the better calculations are reliable to at least about $2 \%$ for M1 transition rates in the ground configurations of B-like and F-like ions, and to about $5 \%$ for the $\mathrm{n}=2$ excited configuration in Be-like ions. The latest such lifetime measurement, from the Heidelberg EBIT, provides a result with an uncertainty as low as $0.22 \%$ for B-like Ar [88]. At this precision, this lifetime result touches on the question whether to use the unscreened or screened electron g-factor in the calculation of the transition rate. Present calculations agree with the measurement only when using the unscreened value, but why this is so, remains under discussion.

\section{E. E1 transitions in Be- to Ne-like Ar}

The $n=2$ intrashell $(\Delta=0)$ electric dipole (E1) transitions of Be- to Ne-like argon ions are in the XUV and 
EUV ranges. Many lines are known (see [4]), but hardly any of them have made it into the NIST collection of consistently precise and reliable data. The interest in these spectral ranges has been revived by recent spacecraft like Chandra and XMM Newton that observe the XUV spectra of many astrophysical objects. However, the understanding of the astrophysical spectra is hampered by inadequate or missing laboratory data to compare with. What an electron beam ion trap can do is illustrated by Fig. 3: a moderate-resolution flat-field grazing incidence spectrometer [91] can fairly quickly produce survey data for a variety of electron energies, and the richness of the spectra that require detailed analysis is evident. By recording overlapping spectra at a range of electron beam energies, but with small energy increments, spectra have been obtained [16] that reveal the rise and fall of individual lines with the respective ion charge state fraction in the trap.

Higher spectral resolution - in principle, when using high-groove density diffraction gratings - is available from generic spectrometers with a narrow entrance slit, like the Schwob-Fraenkel grazing-incidence spectrometer attached to the Berlin EBIT [90]. This instrument employs a concave mirror to image the light source onto the entrance slit of the spectrometer in order to make best use of the available light - after a tedious alignment procedure. Especially for the spectra of highly-charged heavy ions, such an instrument can close long-standing observational gaps. Continuous spectral coverage (in many overlapping exposures) of heavy elements has been demonstrated with this instrument for the wavelength range from about $4 \mathrm{~nm}$ (XUV) to about $100 \mathrm{~nm}$ (VUV).

\section{F. Ne-like Ar IX}

Another route towards higher spectral resolution employs a larger Rowland circle. At Livermore, an $\mathrm{R}=44.3 \mathrm{~m}$ flat-field spectrometer has been built and attached to the electron beam ion trap [65]. This instrument has no physical entrance slit, but relies on imaging the narrow light emission zone onto the detector plane.

Among many others, the spectrum in Fig. 3 shows lines from the $2 \mathrm{p}^{6}-2 \mathrm{p}^{5} 3 \mathrm{~s}, 3 \mathrm{~d}$ transitions in Ne-like Ar IX. Only $\mathrm{J}=1$ levels of the $2 \mathrm{p}^{5} 3 \mathrm{~s}, 3 \mathrm{~d}$ level manifolds can decay to the $\mathrm{J}=0$ ground state by electric dipole (E1) transition. The decay probability of the $2 \mathrm{p}^{5} 3 \mathrm{~s}^{1} \mathrm{P}_{1}^{\mathrm{o}}$ level, in particular in low-charge members of the isoelectronic sequence, is much higher than that of the $2 \mathrm{p}^{5} 3 \mathrm{~s}{ }^{3} \mathrm{P}_{1}^{\mathrm{o}}$ level, because no spin change is needed. Nevertheless, the two levels are intertwined by the mixing due to spin-orbit interaction, which affects the fine structure intervals as well as the level lifetimes. This interaction can be combined into a mixing-angle description [92, 93] that helps to systematize level structure and lifetime data for such pairs of levels.

The spectrum shown here demonstrates something that is qualitatively different from beam-foil data. In the beam-foil light source, observations are time-resolved on the picosecond to nanosecond time scale. Decays of levels with much longer lifetimes are so weak per unit length of observed ion beam that the lines are practically unobservable. In our EBIT-II spectra, however (as mentioned above), we see a second line very close to the $2 \mathrm{p}^{5} 3 \mathrm{~s}{ }^{3} \mathrm{P}_{1}^{\circ}$ level decay at $4.9180 \mathrm{~nm}$ [101] (4.9181 nm [16]). Taking guidance from theory [102], the second line, at $4.9338 \mathrm{~nm}$ [16], is identified with the M2 ground state decay of the $2 \mathrm{p}^{5} 3 \mathrm{~s}{ }^{3} \mathrm{P}_{2}^{\circ}$ level. The remarkable point here is how $\mathrm{E} 1$ and M2 lines appear equally intense in the EUV spectra of the electron beam ion trap. This observation bodes well for the application of electron beam ion trap observations to the interpretation of the new astrophysical XUV data. The new Livermore flat-field spectrometer represents an improvement by a factor of about five in spectral resolution, to be exploited in a working range from 1 to $6 \mathrm{~nm}$. The strong Ar IX lines appear with a single 20-min exposure (Fig. 4).

In the vicinity of these lines, the otherwise strictly forbidden Ar IX line from the $2 \mathrm{p}^{5} 3 \mathrm{~s}{ }^{3} \mathrm{P}_{0}$ level decay to the $\mathrm{J}=0$ ground state has been observed, being induced by the strong magnetic field [94]. Varying the magnetic field strength in the electron beam ion trap between $1 \mathrm{~T}$ and the usual $3 \mathrm{~T}$, the relative intensity of the line changed notably, qualifying this transition for a magnetic field diagnostic.

Further recent (longer-wavelength) EUV data on Ar IX have been reported by Antsiferov et al. from a capillary discharge [95]. Ne-like ions have been studied widely because of their applicability to EUV laser schemes.

In the Ne isoelectronic sequence, the resonance and intercombination (spin-changing) line transition rates for a while seemed settled satisfactorily by a pair of very precise beam-foil lifetime measurements (for $\mathrm{Z}=16,17$ ) [96]. However, later scrutiny of those data in the light of systematic measurements along the isoelectronic sequence [97-99] necessitated massive corrections to those seemingly precise data. The mixing angle analysis of all consistent experimental lifetime data now shows that some older beam-foil lifetime data for $\operatorname{Ar}(Z=18)$ [100] while not being very precise were still more accurate than some later data. The available calculations for the resonance and intercombination line transition probabilities, by the way, scatter by a factor of two.

\section{G. Na-like Ar VIII, Mg-like Ar VII, Al-like Ar VI, Si-like Ar V}

3s-3p transitions in Na-like ions have been intensely studied all across the periodic table of the elements, both for the wavelengths and the transition rates. The wavelengths of the resonance and intercombination transitions in Mg-like Ar VII have been determined at a tokamak discharge and also, taking advantage of a near perfect coincidence of the resonance line in third order of diffraction with the intercombination line in second order, using a 
fast ion beam [8].

The lifetime measurement on the resonance transitions of Na-like Ar VIII [103] fits well into the isoelectronic trend identified by Hutton et al. [104]. The $3 \mathrm{~s}^{2}-3 \mathrm{~s} 3 \mathrm{p}$ transitions in Mg-like ions are much less well covered. For example, no lifetime measurement has yet been done on the $3 \mathrm{~s} 3 \mathrm{p}{ }^{3} \mathrm{P}_{1}^{\circ}$ level of Ar VII (for the corresponding $2 \mathrm{~s} 2 \mathrm{p}{ }^{3} \mathrm{P}_{1}^{\circ}$ level in Be-like Ne VII still only a $50 \%$-estimate based on a plasma observation exists [105]). In fact, no experimental intercombination transition rate data are available for any Be-like ion between $\mathrm{Ne}$ and Fe, nor for any Mg-like ion between $\mathrm{Si}$ and $\mathrm{Fe}$. It is an open question whether such $\mu$ s-range level lifetimes might be better measurable with slow ion beams (discussed in [55]) or with an electron beam ion trap - neither one has been tried yet.

Intercombination transition wavelengths of the Al- and Si-like ions of Ar are known only from an interpolation of (low-q) classical light source and (higher-q) beam-foil data $[7,8]$. A low density light source is needed for the observation, but it ought to incorporate also low energy excitation. Maybe a room temperature ("warm") EBIT with its weaker magnetic field and electron beam of lower density would be appropriate.

\section{H. Rather low charge states of Ar}

The low charge states of argon can be produced and excited in many conventional light sources. However, the number of lines per spectrum in the NIST data base is high only up to Ar III, which reflects the origin of much of the spectroscopic work in the first third of the twentieth century, when light sources generally did not reach higher in charge state. This lack of good data for Ar IV and beyond implies that much more work will be needed to bring the knowledge on the various Ar spectra closer to a practical completion. A typical electron beam ion trap is not particularly well suited for this task as far as relatively low charge states are concerned, and too many charge states have ionization energies below the range of electron beam energies preferred for stable EBIT operation. However, this caveat mostly refers to electron beam ion traps with strong superconducting magnets and their typical dimensions. A warm EBIT with permanent magnets has a lower magnetic field strength and thus compresses the electron beam to a lesser extent. Therefore the electron density in the beam is lower, and the dimensions of the device, in particular the length, are smaller. It might be worthwhile to explore the low charge state operational range of such devices for spectroscopic purposes.

\section{S-like Ar III, Cl-like Ar II}

Among the forbidden (M1) transitions in the ground configuration of S-like Ar III, the $3 \mathrm{~s}^{2} 3 \mathrm{p}^{4}{ }^{1} \mathrm{~S}_{0}$ level lifetime (of order $150 \mathrm{~ms}$ ) has been measured by various ion trap techniques, at long last reaching a precision of just better than 4\% [106]. Unfortunately, electron beam ion traps are not suited for such a study of ions in such low charge states.

In Cl-like ions, the transitions $2 \mathrm{~s}^{2} 2 \mathrm{p}^{5}{ }^{2} \mathrm{P}_{3 / 2,1 / 2}^{\circ}-2 \mathrm{~s} 2 \mathrm{p}^{6}$ ${ }^{2} \mathrm{~S}_{1 / 2}$ can be considered as the resonance transitions. In Ar II, these lines lie in the VUV near 92.0/93.2 nm. Their transition rates have been measured repeatedly by fastion beam techniques two decades ago. The first measurement that reached notable precision in this quest $(1 \%)$, however, is very recent [107]. It employed time-resolved spectroscopy after pulsed ionization and excitation by synchrotron light. No sufficiently good calculation of the transition rates is available yet - the various calculations still differ from each other and from the experimental result by $20 \%$ up to a factor of four (see [108, 109]). Compared to such a theoretical uncertainty, any transition rate data of reasonable reliability are an asset, to be won in the face of rather crowded visible and near-UV spectra that make the analysis of individual decay curves difficult.

Although electron beam ion traps are not particularly suited to trap low-charge state ions like $\mathrm{Ar}^{+}$and $\mathrm{Ar}^{2+}$, the spectra Ar III and Ar II can readily be excited in the single pass of injected Ar atoms through the electron beam, with the trap voltages inverted and thus precluding ion trapping and the production of progressively higher ionization stages. As these calibration lines are useful for the determination of wavelengths in the visible spectrum, which is of interest for a number of applications and physics problems, we discuss the electron beam ion trap observations of this range in a separate section below.

\section{VISIBLE SPECTRA}

The standard reference tables are notably devoid of visible lines associated with highly charged ions, as such energy intervals would either correspond to transitions between high-lying levels (and these would be visible only in the absence of competing decay branches to low-lying levels) or to fine structure intervals, that is to forbidden transitions. The latter have been semiempirically systematized by Kaufman and Sugar [78, 113], but those tables cover only the $n=2$ shell and part of the $n=3$ shell and are incomplete at that.

The first visible range electron beam ion trap spectra of Ar have been obtained at LLNL using a century-old Steinheil-design glass prism spectrometer equipped with a CCD detector. The dispersion was small enough to cover the wavelength range from $450 \mathrm{~nm}$ to the near infrared in only two spectrometer settings, and survey spectra of, for example, $\mathrm{Ne}$ [110], Ar [79, 110], Kr[110], Ba, and Xe [111] were obtained for a series of closely spaced electron beam energies. The appearance thresholds of certain lines in these spectra revealed their origin with 
specific high charge state ions, and a number of M1 transitions could thus be recognized and identified (see also Fig. 2).

In Ar spectra from electron beam ion traps that are recorded with higher resolution, many weak lines show most of which remain unidentified. The spatial emission of these lines indicates that they originate from the geometrical overlap zone of the ballistically injected gas beam (under ultrahigh vacuum conditions) and the electron beam. They do not show the trap-filling pattern of emission from highly charged ions. In fact, the drift tube voltages can be changed to prevent trapping ('inverted trap' [112]), and even then these lines appear, indicating that they must originate from low charge states that are reached in a single pass through the electron beam. These lines make for excellent calibrations of electron beam ion trap spectra, by alternating recordings with the drift tubes in regular and inverted mode - if they can be identified with some lines in spectral tables that carry exceedingly precise wavelength values from measurements in the $1930 \mathrm{~s}$. Note, however, that many of these precisely determined lines have never been classified. A practical problem lies in the fact that relative line intensities are so different among light sources that it is difficult to recognize the well established lines among the many.

Interspersed between those lines from low-charge ions appear a number of fairly intense lines which by their emission from the whole trap length can be judged to originate from high-charge state ions. Also, their emission zone is wider than the electron beam, because highly charged ions are better bound by the trap and thus can remain trapped at higher ion temperatures. At the LLNL electron beam ion trap, a fast transmission-grating spectrometer (TGS) has been employed for high-resolution observations in the visible [89]. The spectra demonstrate that this line broadening may be exploited as a tool that singles out emissions from long-lived levels of highly charged ions. The same spectrometer was used in combination with the inverted-trap in situ calibration technique in the measurement of ground-state hyperfine transitions in H-like thallium (Z=82), $\mathrm{Tl}^{81+}[116]$.

\section{CONCLUSION AND OUTLOOK}

Considering that quantum mechanics is available as a tool for the understanding of atomic structure since about 80 years, it is remarkable to see that there are 70 year old precision measurements of, for example, Ar spectra that as yet defy line classification, even knowing that the respective charge states must be fairly low.

Later developments of light sources have made all charge states of Ar accessible to experiment. Many of the spectra are simpler for the ions with fewer electrons, but the demands on precision and accuracy are higher when trying to extract details of the atomic structure and physics. For the few-electron ions, experiment seems to be more seriously challenged than theory. For all but the highest charge states, line identification is grossly incomplete. Even for He-like Ar, there are transitions between relatively low-lying levels that are still being disputed.

Traditionally less well studied than the other ranges is the EUV, because of the low efficiency of reflective optics and the shortage of reference lines. The Livermore flat-field spectrometer measurements clearly demonstrate that the light emission from an electron beam ion trap is sufficient for precision spectrometry in this range, too. The high spectral resolution achieved matches the needs in understanding the many details of laboratory and astrophysical (Chandra and XMM Newton space craft) data in the wavelength range below $10 \mathrm{~nm}$. Also, time-resolved measurement techniques deserve to be developed in order to identify and investigate specific levels in a variety of ions that must radiate in astrophysical light sources but remain presently unidentified for lack of laboratory data.

\section{Acknowledgment}

The author appreciates helpful comments on the manuscript made by Indrek Martinson (Södra Sandby) and Peter Beiersdorfer (Livermore). He gratefully acknowledges travel support from the German Research Association (DFG), financial support by FNRS (Belgium) and NSERC (Canada) as well as the hospitality experienced in the LLNL electron beam ion trap group. The work at University of California Lawrence Livermore National Laboratory was performed under the auspices of the Department of Energy under Contract No. W-7405Eng-48.
[1] Eidelsberg, M., Crifo-Magnant, F. and Zeippen, C. J., Astron. Astrophys. Suppl. Ser. 43, 455 (1981).

[2] Edlén, B., Phys. Scr. T 8, 5 (1984).

[3] In the World Wide Web at http://Physics.nist.gov/PhysRefData/contents.html

[4] Kelly, R. L., atomic spectra data collection as maintained on the WWW at http://cfa- www.harvard.edu/amdata/ampdata/kelly/kelly.html

[5] Lesteven-Vä̈sse, I., Chantepie, M., Grandin, J. P., Hennecart, D., Husson, X., Lecler, D., Buchet, J. P., Buchet-Poulizac, M. C., Désesquelles, J. and Martin, S., Physica Scripta 34, 138 (1986).

[6] Lesteven-Vaisse, I., Folkmann, F., Ben Sitel, A., Chantepie, M. and Lecler, D., Physica Scripta 38, 45 
(1988).

[7] Träbert, E., Heckmann, P. H., Hutton, R. and Martinson, I., J. Opt. Soc. Am. B 5, 2173 (1988).

[8] Träbert, E., Hutton, R., Engström, L, Bliman, S., Berry, H. G., Kurtz, C., Phys. Lett. A 129, 381 (1988).

[9] Levine, M. A., Marrs, R. E., Henderson, J. R., Knapp, D. A. and Schneider, M. B., Physica Scripta T 22, 157 (1988).

[10] Levine, M. A., Marrs, R. E., Bardsley, J. N., Beiersdorfer, P., Bennett, C. L., Chen, M. H., Cowan, T., Dietrich, D., Henderson, J. R., Knapp, D. A., Osterheld, A., Penetrante, B. M., Schneider, M. B. and Scofield, J. H., Nucl. Instrum. Meth. B 43, 431 (1989).

[11] Gould, H., Greiner, D., Lindstrom, P., Symons, T. J. M. and Crawford, H., Bull. Am. Phys. Soc. 28, 1345 (1983).

[12] Träbert, E., Beiersdorfer, P., Utter, S. B. and Crespo López-Urrutia, J.R., Physica Scripta 58, 599 (1998).

[13] Utter, S. B., Beiersdorfer, P., Crespo López-Urrutia, J R. and Widmann, K., Nucl. Instrum. Meth. A 428, 276 (1999).

[14] Schweikhard, L., Beiersdorfer, P., Brown, G. V., Crespo López-Urrutia, J. R., Utter, S. B. and Widmann, K., Nucl. Instrum. Meth. Phys. Res. B 142, 245 (1998).

[15] Lepson, J. K., Beiersdorfer, P., Brown, G. V., Kahn, S M., Liedahl, D. A., Mauche, C. W. and Utter, S. B., Rev. Mex. A. A. (Serie de Conferencias) 9, 137 (2000).

[16] Lepson, J. K., Beiersdorfer, P., Behar, E., and Kahn, S. M., Astrophys. J. 590, 604 (2003).

[17] Träbert, E., Beiersdorfer, P. and Utter, S. B. Physica Scripta T 80, 450 (1999).

[18] Beiersdorfer, P., Schweikhard, L., Crespo LópezUrrutia, J. and Widmann, K., Rev. Sci. Instrum. 67, 3818 (1996)

[19] Träbert, E., Beiersdorfer, P., Utter, S. B., Brown, G. V., Chen, H., Harris, C. L., Neill, P. A., Savin, D. W. and Smith, A. J., Astrophys. J. 541, 506 (2000).

[20] Träbert, E. and Beiersdorfer, P., Rev. Sci. Instrum. 74, 2127 (2003)

[21] Beiersdorfer, P., Beck, B., Becker, St., and Schweikhard, L., Int. J. of Mass Spectrometry and Ion Processes 157/158, 1491 (1996).

[22] Beiersdorfer, P., Osterheld, A. L., Decaux, V. and Widmann, K., Phys. Rev. Lett. 77, 5353 (1996).

[23] Gould, H. and Marrus, R., Phys. Rev. A 28, 2001 (1984).

[24] Briand, J. P., Tavernier, M., Indelicato, P., Marrus, R. and Gould, H., Phys. Rev. Lett. 50, 832 (1983).

[25] Briand, J. P., Mossé, J. P., Indelicato, P., Chevallier, P., Girard-Vernhet, D., Chetioui, A., Ramos, M. T. and Desclaux, J. P., Phys. Rev. A 28, 1413 (1983).

[26] Beyer, H. F., Mokler, P. H., Deslattes, R. D., Folkmann, F. and Schartner, K.-H., Z. Phys. A 318, 249 (1984).

[27] Beyer, H. F., Deslattes, R. D., Folkmann, F. and La Villa, R. E., J. Phys. B 18, 207 (1985).

[28] Marmar, E. S., Rice, J. E., Källne, K., Källne, E. and La Villa, R. E., Phys. Rev. A 33, 774 (1986).

[29] Klöpfel, D., Hölzer, G., Förster, E. and Beiersdörfer, P., Rev. Sci. Instrum. 68, 3669 (1997)

[30] Hölzer, G., Förster, E., Klöpfel, D., Beiersdorfer, P., Brown, G. V., Crespo López-Urrutia, J. R. and Widmann, K., Phys. Rev. A 57, 945 (1998).

[31] Tschischgale, J., Klöpfel, D., Beiersdorfer, P., Brown, G. V., Förster, E., Schulte-Schrepping, H. and Utter, S.
B., Can. J. Phys. 80, 867 (2002).

[32] Johnson, W. R. and Soff, G., At. Data Nucl. Data Tables 33, 405 (1985).

[33] Schweppe, J., Belkacem, A., Blumenfeld, L., Claytor, N., Feinberg, B., Gould, H., Kostroun, V. E., Levy, L., Misawa, S., Mowat, J. R., Prior, M. H., Phys. Rev. Lett. 66, 1436 (1991).

[34] Brandau, C., Kozhuharov, C., Müller, A., Shi, W., Schippers, S., Bartsch, T., Bhm, S., Bhme, C., Hoffknecht, A., Knopp, H., Grn, N., Scheid, W., Steih, T., Bosch, F., Franzke, B., Mokler, P. H., Nolden, F., Steck, M., Stöhlker, T., and Stachura, Z., Phys. Rev. Lett. 91, 073202 (2003).

[35] Beiersdorfer, P. (private communication).

[36] Müller, D., Gassen, J., Kremer, L., Pross, H.-J., Scheuer, F., Sträter, H.-D., von Brentano, P., Pape, A., and Sens, J. C.. Europhys. Lett. 5, 503 (1988).

[37] Silver, J. D., Varney, A. J., Margolis, H. S., Baird, P. E. G., Grant, I. P., Groves, P. D., Hallett, W. A., Handford, A. T., Hirst, P. J., Holmes, A. R., Howie, D. J. H. Hunt, R. A., Nobbs, K. A., Roberts, M., Studholme, W., Wark, J. S., Williams, M. T., Levine, M. A., Dietrich, D. D., Graham, W. G., Williams, I. D., and O'Neil, R., Rev. Sci. Instrum. 65, 1072 (1994).

[38] Gabriel, A. H., Mon. Not. R. Astron. Soc. 160, 99 (1972).

[39] Tarbutt, M. R., Barnsley, M. R., Peacock, N. J. and Silver, J. D., J. Phys. B: At. Mol. Opt. Phys. 343979 (2001).

[40] Currell, F. J., Asada, J., Back, T. V., Dong, C. Z., Margolis, H. S., Nakamura, N., Ohtani, S., Silver, J. D., Watanabe, H., J. Phys. B 33, 727 (2000).

[41] Beyer, H. F., Folkmann, F. and Schartner, K.-H., Z. Phys. D 19, 65 (1986).

[42] Hallett, W. A., Howie, D. J. H., Silver, J. D. and Dietrich, D. D., Phys. Lett. A 192, 43 (1994).

[43] Livingston, A. E., Kukla, K. W., Vogel Vogt, C. M., Berry, H. G., Dunford, R. W., Gemmell, D. S., Kanter, E. P., Suleiman, J., Ali, R., Cheng, S. and Curtis, L. J., Nucl. Instrum. Meth. Phys. Res. B 98, 28 (1995).

[44] Beiersdorfer, P., von Goeler, S., Bitter, M. and Hill, K. W., Nucl. Instrum. Meth. Phys. Res. B 43, 431 (1989).

[45] Beiersdorfer, P., Osterheld, A. L., Phillips, T. W., Bitter, M., Hill, K. W. and von Goeler, S., Phys. Rev. E 52, 1980 (1995).

[46] Beiersdorfer, P., Crespo López-Urrutia, J., Decaux, V., Widmann, K. and Neill, P., Rev. Sci. Instrum. 68, 1073 (1997).

[47] Wong, K. L., Beiersdorfer, P., Reed, K. J. and Vogel, D. A., Phys. Rev. A 51, 1214 (1995).

[48] Smith, A. J., Beiersdorfer, P., Decaux, V., Widmann, K., Reed, K. J. and Chen, M. H., Phys. Rev. A 54, 462 (1996).

[49] Jacobs, V. L.. Decaux, V. and Beiersdorfer, P., J. Quant. Spect. Rad. Transf. 58, 645 (1997).

[50] Antsiferov, P. S. and Churilov, S. S., Physica Scripta 64, 292 (2001).

[51] Biedermann, C., Radtke, R. and Fournier, K. B., Phys. Rev. E 66, 066404 (2002).

[52] Beiersdorfer, P., Bitter, M., Hey, D. and Reed, K.J., Phys. Rev. A 66, 032504 (2002).

[53] Dohmann, H. and Mann, R., Z. Physik A 291, 15 (1979).

[54] Gabriel, A. H. and Jordan, C., Nature 221, 947 (1969). 
[55] Träbert, E., in "Accelerator-based Atomic Physics Techniques and Applications", (Edited by S. M. Shafroth and J. C. Austin) (Washington: Am. Inst. Phys.) 1997, p. 567

[56] Träbert, E., Nucl. Instrum. Meth. Phys. Res. B 98, 10 (1995).

[57] Träbert, E., Physica Scripta 61, 257 (2000).

[58] Träbert, E., Can. J. Phys. 80, 1481 (2002).

[59] Crespo López-Urrutia, J. R., Beiersdorfer, P., Savin, D. W. and Widmann, K., Phys. Rev. A 57, 238 (1998)

[60] Träbert, E., Beiersdorfer, P., Brown, G. V., Smith, A. J., Utter, S. B., Gu, M. F. and Savin, D. W., Phys. Rev. A 60, 2034 (1999).

[61] Marrus, R., and Schmieder, R.W., Phys. Rev. Lett. 25, 1689 (1970).

[62] Hubricht, G. H. and Träbert, E., Z. Phys. D 7, 243 (1987).

[63] Kentsch, U., Zschornack, G., Grossmann, F., Ovsyannikov, V. P. and Ullmann, F., Nucl. Instrum. Meth. Phys. Res. B 205, 266 (2003).

[64] Biedermann, C., Radtke, R. and Fournier, K., Nucl. Instrum. Meth. Phys. Res. B 205, 255 (2003).

[65] Beiersdorfer, P., Magee, E. W., Träbert, E., Chen, H., Lepson, J. K., Gu, M.-F., Schmidt, M., Rev. Sci. Instrum. 75, 3723 (2004).

[66] Edlén, B., Physica Scripta 19, 255 (1979).

[67] Widing, K. G. and Purcell, J. D., Astrophys. J. 204, L151 (1976).

[68] Kim, Y.-K., Baik, D. H., Indelicato, P., and Desclaux, J. P., Phys. Rev. A 44, 148 (1991).

[69] Träbert, E., Doerfert, J., Granzow, J., Büttner, R., Brauckhoff, J., Nicolai, M., Schartner, K.-H., Folkmann, F. and Mokler, P.H., Phys. Lett. A 202, 91 (1995).

[70] Zou, Y., Hutton, R., Feili, D., Neacsu, C., Ma, X., Schartner, K.-H., Mokler, P. H., Nucl. Instrum. Meth. Phys. Res. B (to be published).

[71] Smitt, R., Solar Physics 51, 113 (1977).

[72] Berry, H. G., Désesquelles, J., Cheng, K. T. and Schectman, R. M., Phys. Rev. A 8, 546 (1978).

[73] Tunnell, T. W. and Bhalla, C. P., Phys. Lett. 72 A, 19 (1979).

[74] Safronova, U. I., Johnson, W. R. and Derevianko, A., Physica Scripta 60, 46 (1999).

[75] Edlén, B., Optica Pura y Aplicada 10, 123 (1977).

[76] Edlén, B., Physica Scripta 23, 1079 (1981).

[77] Edlén, B., Physica Scripta 28, 51 (1983).

[78] Kaufman, V. and Sugar, J., J. Phys. Chem. Ref. Data 15, 321 (1986).

[79] Crespo López-Urrutia, J. R., Beiersdorfer, P., Widmann, K. and Decaux, V., in "Nineteenth International Conference on the Physics of Electronic and Atomic Collisions - Scientific Program and Abstracts of Contributed Papers", (Edited by J. B. A. Mitchell, J. W. McConkey and C. E. Brion) (ICPEAC, Whistler, BC, 1995), p. 589.

[80] Bieber, D. J., Margolis, H. S., Oxley, P. K. and Silver, J. D., Phys. Scr. T 73, 64 (1997).

[81] Back, T. V., Margolis, H. S., Oxley, P. K., Silver, J. D. and Myers, E. G. Hyperfine Int., 114, 203 (1998).

[82] Serpa, F. G., Gillaspy, J. D. and Träbert, E., J. Phys. B: At. Mol Opt. Phys. 31, 3345 (1998).

[83] Moehs, D. P. and Church, D. A., Phys. Rev. A 58, 1111 (1998).

[84] Draganić, I., Crespo López-Urrutia, J. R., DuBois, R.,
Fritzsche, S., Shabaev, V. M., Soria Orts, R., Tupitsyn, I. I., Zou, Y., Ullrich, J., Phys. Rev. Lett, 91, 183001 (2003).

[85] Träbert, E., Gwinner, G., Wolf, A., Tordoir, X. and Calamai, A. G., Phys. Lett. A 264, 311 (1999).

[86] Träbert, E., Beiersdorfer, P., Brown, G. V., Chen, H., Pinnington, E. H. and Thorn, D. B., Phys. Rev. A 64, 034501 (2001).

[87] Träbert, E., Beiersdorfer, P., Gwinner, G., Pinnington, E. H. and Wolf, A., Phys. Rev. A 66, 052507 (2002).

[88] Crespo López-Urrutia, J.R., Braun, J., Brenner, G., Bruhns, H., Dimopoulou, C., Draganić, I. N., Fischer, D., González-Martínez, A. J., Lapierre, A., Mironov, V., Moshammer, R., Soria Orts, R., Tawara, H., Trinczek, M., and Ullrich, J., J. Phys.: Conf. Ser. 2, 42 (2004).

[89] Utter, S. B., Crespo López-Urrutia, J. R., Beiersdorfer, P. and Träbert, E., Rev. Sci. Instrum. 73, 3737 (2002).

[90] Biedermann, C., Radtke, R., Schwob, J.-L., Mandelbaum, P., Doron, R., Fuchs, T. and Fußmann, G., Phys. Scr. T 9285 (2001).

[91] Utter, S. B., Beiersdorfer, P., Crespo López-Urrutia, J. R. and Träbert, E., Rev. Sci. Instrum. 70, 288 (1999).

[92] Curtis, L. J., J. Phys. B: Atom. Mol. Opt. Phys. 25, $1425(1992)$

[93] Curtis, L. J., Physica Scripta 48, 559-66 (1993).

[94] Beiersdorfer, P., Scofield, J. J., and Osterheld, A. L., Phys. Rev. Lett. 90, 235003 (2003).

[95] Antsiferov, P. S., Churilov, S. S., Dorokhin, L. A., Koshelev, K. N., Nazarenko, A. V. and Sidelnikov, Yu. V., Physica Scripta 62, 127 (2000).

[96] Westerlind, M., Engström, L., Bengtsson, P. and Curtis, L. J., Phys. Rev. A 45, 6198 (1992).

[97] Curtis, L. J., Maniak, S. T., Ghrist, R. W., Irving, R. E., Ellis, D. G., Henderson, M., Kacher, M. H., Träbert, E., Granzow, J., Bengtsson, P. and Engström, L., Phys. Rev. A 51, 4575 (1995).

[98] Träbert, E., Physica Scripta 53, 167 (1996).

[99] Kirm, M., Bengtsson, P. and Engström, L., Phys. Scr. 54, 167 (1996).

[100] Berry, H. G., Désesquelles, J., Cheng, K. T. and Schectman, R. M., Phys. Rev. A 18, 546 (1978).

[101] Phillips, L. W. and Parker, W. L., Phys. Rev. 60, 301 (1941).

[102] Hibbert, A., Le Dourneuf, M. and Mohan, M., At. Data Nucl. Data Tables 53, 23 (1993).

[103] Reistad, N., Engström, L. and Berry, H. G., Phys. Scr. 34, 158 (1986).

[104] Hutton, R., Engström, L. and Träbert, E., Phys. Rev. Lett. 60, 2469 (1988).

[105] Kunze, H. J., Space Sci. Rev. 13, 565 (1972).

[106] Träbert, E. and Gwinner, G., Phys. Rev. A 65, 014501 (2002).

[107] Lauer, S., Liebel, H., Vollweiler, F., Schmoranzer, H., Lagutin, B. M., Demekhin, Ph. V., Petrov, I. D. and Sukhorukov, V. L., J. Phys B: At. Mol. Opt. Phys. 32, 2015 (1999).

[108] Biémont, E. and Träbert, E., J. Phys. B: At. Mol. Opt. Phys. 33, 2939 (2000).

[109] Berrington, K. A., Pelan, J. C. and Waldock, J. A., J. Phys. B: At. Mol. Opt. Phys. 34, L419 (2001).

[110] Chen, H., Beiersdorfer, P., Harris, C. L., Träbert, E., Utter, S. B. and Wong, K. L., Physica Scripta T 92, $284(2001)$

[111] Crespo López-Urrutia, J. R., Beiersdorfer, P., Wid- 
mann, K. and Decaux, V., Physica Scripta T 80, 448 (1999).

[112] Chen, H., Beiersdorfer, P., Harris, C. L., Träbert, E., Utter, S. B. and Wong, K. L., Physica Scripta T 92, 284 (2001).

[113] Sugar, J. and Kaufman, V., J. Opt. Soc. Am. B 1, 218 (1984).

[114] Edlén, B., Z. Astrophysik 22, 30 (1942).

[115] Utter, S. B., Beiersdorfer, P., Brown, G. V., Clothiaux, E. J. and Podder, N. K., Rev. Sci. Instrum. 70, 284 (1999).

[116] Beiersdorfer, P., Utter, S. B., Wong, K. L., Crespo López-Urrutia, J. R., Britten, J. A., Chen, H., Harris, C. L., Thoe, R. S., Thorn, D. B., Träbert, E., Gustavsson, M. G. H. and Forssén, C., Mårtensson-Pendrill, A.-M., Phys. Rev. A 64, 032506 (2001). 


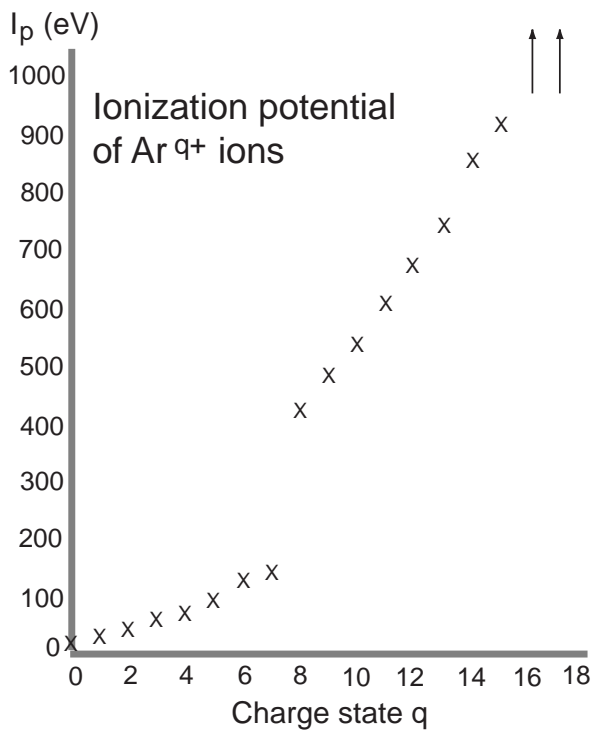

FIG. 1: Ionization potentials of Ar ions. The K-shell ionization energies of He-like $\mathrm{Ar}^{16+}$ and H-like $\mathrm{Ar}^{17+}$ ions amount to several $\mathrm{keV}$. In an electron beam ion trap with an electron beam energy of about $400 \mathrm{eV}$ (which is low for typical operations), all low charge state ions are rapidly ionized up to $\mathrm{q}=8+$.

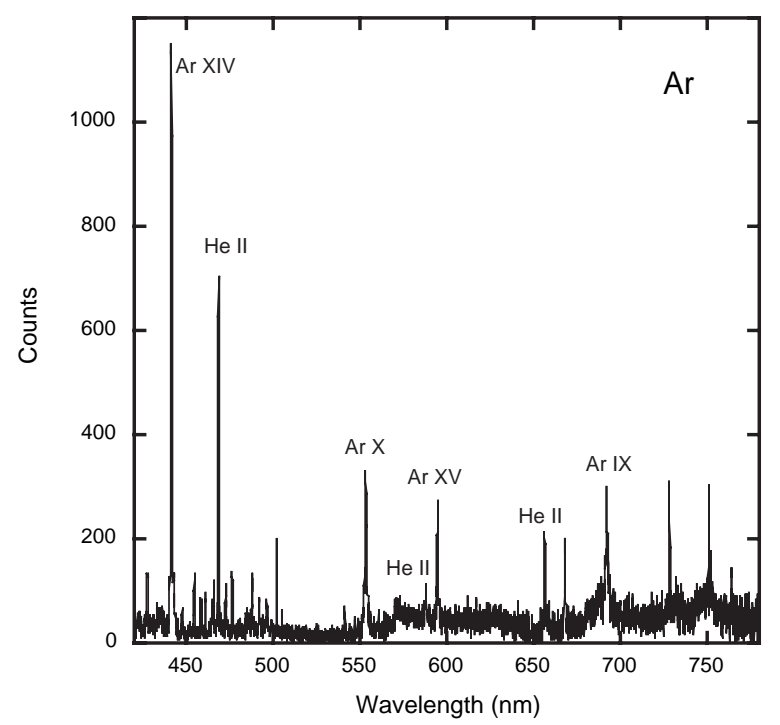

FIG. 2: Survey spectrum or Ar in the visible range. The spectrum has been composed of overlapping individual sections of $80 \mathrm{~nm}$ spectra width that have been recorded with a 1 -m normal-incidence spectrometer using a $300 \mathrm{l} / \mathrm{mm}$ grating and a CCD detector [91]. All lines with a visibly broad foot arise from M1 transitions. 


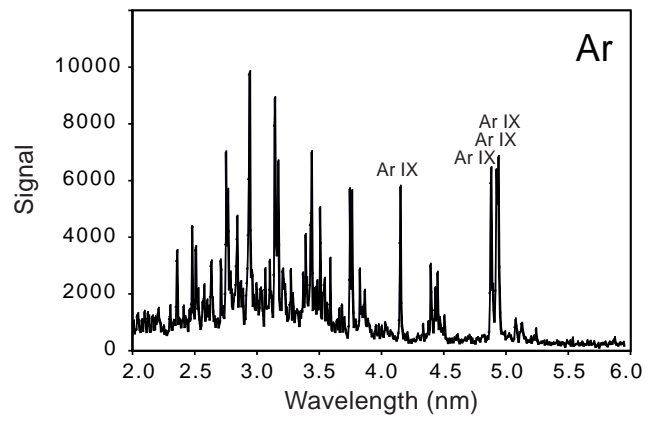

FIG. 3: Extreme-UV spectrum of Ar in the electron beam ion trap, recorded by a flat-field spectrometer [115] equipped with a CCD camera. The marked Ar IX lines are from $2 \mathrm{p}^{6}-$ $2 \mathrm{p}^{5} 3 \mathrm{l}$ transitions.

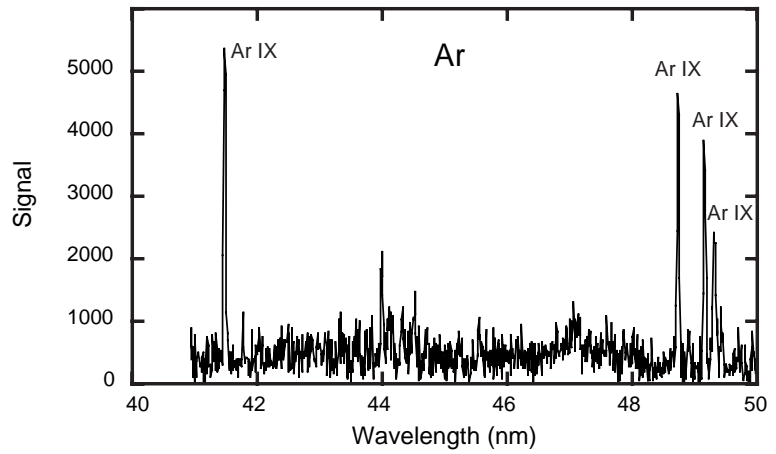

FIG. 4: Extreme-UV spectrum of Ar in the electron beam ion trap, recorded by a high-resolution flat-field spectrometer equipped with a CCD camera. The marked Ar IX lines are from $2 p^{6}-2 p^{5} 31$ transitions. Compare the resolution to the spectrum in Fig. 3. 